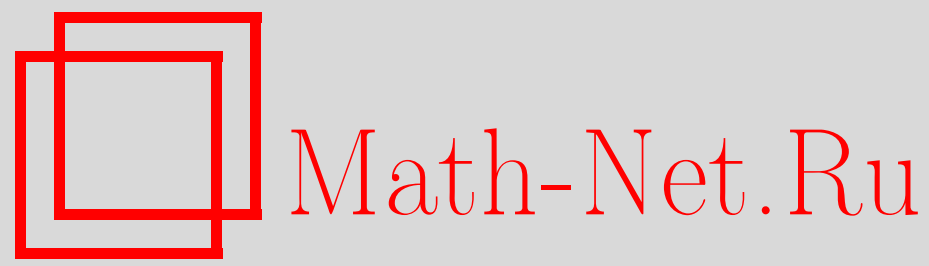

А. В. Томилин, Кусочно-параболические сплайнкривые с узлами в вершинах выпуклого четырехугольника, Матем. заметки, 1996, том 60, выпуск 3, 474-477

DOI: https://doi.org/10.4213/mzm1855

Использование Общероссийского математического портала MathNet.Ru подразумевает, что вы прочитали и согласны с пользовательским соглашением

http://www . mathnet.ru/rus/agreement

Параметры загрузки:

IP: 34.229 .108 .108

26 апреля 2023 г., 11:41:10

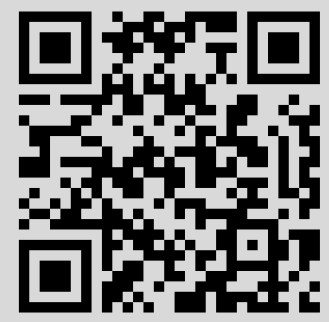




\section{КУСОЧНО-ПАРАБОЛИЧЕСКИЕ СПЛАЙН-КРИВЫЕ С УЗЛАМИ В ВЕРШИНАХ ВЫПУКЛОГО ЧЕТЫРЕХУГОЛЬНИКА}

\section{А. В. Томилин}

Пусть $Q\left(\Delta\right.$ - класс замкнутых кусочно-параболических кривых гладкости $C^{2}$ по натуральному параметру без самопересечений с множеством узлов $\Delta, \Delta=\left\{f_{1}, \ldots\right.$, $\left.f_{n}\right\}, f_{i} \in \mathbb{R}^{2}, f_{i} \neq f_{i+1}$, при $i=\overline{1, n}, f_{n+1}=f_{1}$. Класс $Q(\Delta)$ был рассмотрен в статье [1]. Доказательство существования кривых из $Q(\Delta)$, где $\Delta$ - набор вершин неправильного вьпуклого четырехугольника, в [1] дано не было и будет изложено здесь. Вопрос о единственности в этом случае остается открытым.

Пусть $f_{1} f_{2} f_{3} f_{4}$ - выпукльй четырехугольник, $\Delta=\left\{f_{1}, f_{2}, f_{3}, f_{4}\right\}, \gamma_{i}$ - внешний угол при вершине $f_{i}, h_{i}=\left|f_{i} f_{i+1}\right|, \alpha=\left(\alpha_{1}, \alpha_{2}, \alpha_{3}, \alpha_{4}\right), \alpha_{i} \in\left(0, \gamma_{i}\right), \alpha_{i}+$ $\gamma_{i+1}-\alpha_{i+1}<\pi, i=\overline{1,4}, f_{5}=f_{1}, \alpha_{5}=\alpha_{1}, \gamma_{5}=\gamma_{1}$. Набор $\alpha$ однозначно определяет замкнутую кусочно-параболическую кривую $p(\alpha, \Delta)$ гладкости $C^{1}$ по натуральному параметру с множеством узлов $\Delta$ такую, что угол между касательной к кривой в узле $f_{i}$ и стороной $f_{i} f_{i+1}$, отсчитываемый от стороны, равен $\alpha_{i}$. Обозначим: $\operatorname{cur}_{i}(\alpha, \Delta), \operatorname{cur}_{i}^{-}(\alpha, \Delta)$ - кривизны кривой $p(\alpha, \Delta)$ в узле $f_{i}$ справа и слева соответственно $i=\overline{1,4}$. Кривизны определяются по формулам:

$$
\begin{aligned}
\operatorname{cur}_{i}(\alpha, \Delta) & =\frac{1}{2 h_{i}}\left(\operatorname{ctg} \alpha_{i}+\operatorname{ctg}\left(\gamma_{i+1}-\alpha_{i+1}\right)\right)^{2} \sin ^{3} \alpha_{i}, \\
\operatorname{cur}_{i}^{-}(\alpha, \Delta) & =\frac{1}{2 h_{i-1}}\left(\operatorname{ctg} \alpha_{i-1}+\operatorname{ctg}\left(\gamma_{i}-\alpha_{i}\right)\right)^{2} \sin ^{3}\left(\gamma_{i}-\alpha_{i}\right) .
\end{aligned}
$$

Существование кривых из $Q(\Delta)$ достаточно доказать с точностью до аффиннных преобразований, так как при афффинных преобразованиях гладкость не нарушается и параболы переходят в параболы.

Любой выпуклый четырехугольник можно афффинными преобразованиями привести к виду $f_{1}^{0} f_{2}^{0} f_{3}^{0} f_{4}^{0}$ :

$$
\begin{gathered}
\Delta^{0}=\left\{f_{1}^{0}, f_{2}^{0}, f_{3}^{0}, f_{4}^{0}\right\}, \quad \gamma_{1}^{0} \in\left[\frac{\pi}{2} ; \frac{3 \pi}{4}\right), \quad \gamma_{2}^{0}=\frac{\pi}{2}, \quad \gamma_{3}^{0} \in\left[\frac{\pi}{2} ; \frac{3 \pi}{4}\right), \quad \gamma_{4}^{0} \in\left(0 ; \frac{\pi}{2}\right], \\
h_{1}^{0}=h_{2}^{0}=1, \quad h_{3}^{0} \in(0 ; \sqrt{2}), \quad h_{4}^{0} \in(0 ; \sqrt{2}) .
\end{gathered}
$$

Выберем точку $f$ так, что $f_{1}^{0} f_{2}^{0} f_{3}^{0} f$ будет квадратом. Если $f_{4}^{0}=f$, то класс $Q\left(\Delta^{0}\right)$ не пуст и состоит из единственной кривой (это доказано в [1]).

Если $f_{4}^{0} \neq f$, то параметризуем сегмент $f_{4}^{0} f: \varphi(t)=(1-t) f_{4}^{0}+t f, t \in[0 ; 1]$, $\varphi:[0 ; 1] \rightarrow \mathbb{R}^{2}$.

Для $\Delta(t)=\left\{f_{1}^{0}, f_{2}^{0}, f_{3}^{0}, \varphi(t)\right\}, t \in[0 ; 1]$, имеем

$$
\begin{gathered}
\gamma_{1}(t) \in\left[\frac{\pi}{2} ; \gamma_{1}^{0}\right], \quad \gamma_{2}(t)=\frac{\pi}{2}, \quad \gamma_{3}(t) \in\left[\frac{\pi}{2} ; \gamma_{3}^{0}\right], \quad \gamma_{4}(t) \in\left[\gamma_{4}^{0} ; \frac{\pi}{2}\right], \\
h_{1}(t)=h_{2}(t)=1, \quad h_{3}(t) \in[a ; \sqrt{2}), \quad h_{4} \in[a ; \sqrt{2}), \quad a=\min \left\{h_{3}^{0}, h_{4}^{0}\right\} .
\end{gathered}
$$


Положим $\omega_{\varepsilon}(t)=\left\{\alpha \mid \alpha_{i} \in\left(\varepsilon ; \gamma_{i}(t)-\varepsilon\right), \alpha_{i}+\gamma_{i+1}-\alpha_{i+1}<\pi, i=\overline{1,4}\right\}$, где $\varepsilon$ - малое положительное число, $t \in[0 ; 1]$;

$$
F: \mathbb{R}^{5} \rightarrow \mathbb{R}^{4}, \quad F(\alpha, t)=\left(F_{1}(\alpha, t), F_{2}(\alpha, t), F_{3}(\alpha, t), F_{4}(\alpha, t)\right),
$$

где $F_{i}(\alpha, t)=\operatorname{cur}_{i}(\alpha, \Delta(t))-\operatorname{cur}_{i}^{-}(\alpha, \Delta(t)), i=\overline{1,4}, t \in[0 ; 1]$.

Лемма. Существует постоянная $\varepsilon_{0}, \varepsilon_{0}>0$, такая, что для любого $\varepsilon$ из $\left(0 ; \varepsilon_{0}\right]$ и для любого $t$ из $[0 ; 1]$ верно: если $\alpha \in \partial \omega_{\varepsilon}(t)$, то $F(\alpha, t) \neq 0$.

ДОКАЗАТЕЛЬСТво. а) Пусть $\alpha_{i}+\gamma_{i+1}(t)-\alpha_{i+1}=\pi$ для некоторого $i, 1 \leqslant i \leqslant 4$, и некоторого $t, t \in[0 ; 1]$, тогда $\operatorname{cur}_{i}(\alpha, \Delta(t))=0$.

С другой стороны,

$$
\alpha_{i}>\pi-\gamma_{i+1}(t), \quad \alpha_{i-1}+\gamma_{i}(t)-\alpha_{i}<\gamma_{i-1}(t)+\gamma_{i}(t)+\gamma_{i+1}(t)-\pi<\pi,
$$

откуда следует, что $\operatorname{cur}_{i}^{-}(\alpha, \Delta(t)) \neq 0, F_{i}(\alpha, t) \neq 0, F(\alpha, t) \neq 0$.

б) Пусть $\alpha_{1}=\varepsilon$ для некоторого $t, t \in[0 ; 1]$. Предположим, что $F(\alpha, t)=0$. Из предположения, в частности, следует:

$$
\begin{gathered}
\operatorname{cur}_{2}(\alpha, t)=\operatorname{cur}_{2}^{-}(\alpha, t), \\
\left(\operatorname{ctg} \alpha_{2}+\operatorname{ctg}\left(\gamma_{3}(t)-\alpha_{3}\right)\right)^{2} \sin ^{3} \alpha_{2}=\left(\operatorname{ctg} \varepsilon+\operatorname{tg} \alpha_{2}\right)^{2} \cos ^{3} \alpha_{2}, \\
\operatorname{ctg}\left(\gamma_{3}(t)-\alpha_{3}\right)=\operatorname{ctg} \alpha_{2}\left(\operatorname{ctg} \varepsilon \operatorname{ctg}^{1 / 2} \alpha_{2}+\operatorname{tg}^{1 / 2} \alpha_{2}-1\right),
\end{gathered}
$$

откуда для достаточно малых $\varepsilon$ имеем

$$
\operatorname{ctg}\left(\gamma_{3}(t)-\alpha_{3}\right)>\operatorname{ctg} \alpha_{2}, \quad \gamma_{3}(t)-\alpha_{3}<\alpha_{2},
$$

причем выбор $\varepsilon$ не зависит от $t$.

С другой стороны,

$$
\operatorname{cur}_{1}(\alpha, \Delta(t))=\frac{1}{2}\left(\operatorname{ctg} \varepsilon+\operatorname{tg} \alpha_{2}\right)^{2} \sin ^{3} \varepsilon=\frac{1}{2}\left(\cos \varepsilon+\sin \varepsilon \operatorname{tg} \alpha_{2}\right)^{2} \sin \varepsilon,
$$

$\operatorname{cur}_{1}(\alpha, \Delta(t)) \asymp \varepsilon$ для малых $\varepsilon$ (независимо от $t$ ).

По предположению $\operatorname{cur}_{1}(\alpha, \Delta(t))=\operatorname{cur}_{1}^{-}(\alpha, \Delta(t))$, следовательно, для малых $\varepsilon$ $\operatorname{cur}_{1}^{-}(\alpha, \Delta(t)) \asymp \varepsilon$,

$$
\begin{aligned}
\operatorname{cur}_{1}^{-}(\alpha, \Delta(t)) & =\frac{1}{2 h_{4}(t)}\left(\operatorname{ctg} \alpha_{4}+\operatorname{ctg}\left(\gamma_{1}(t)-\varepsilon\right)\right)^{2} \sin ^{3}\left(\gamma_{1}(t)-\varepsilon\right) \\
& =\frac{\sin ^{2}\left(\alpha_{4}+\gamma_{1}(t)-\varepsilon\right) \sin \left(\gamma_{1}(t)-\varepsilon\right)}{2 h_{4}(t) \sin ^{2} \alpha_{4}},
\end{aligned}
$$

откудаполучаем для малых $\varepsilon \pi-\gamma_{1}(t)-\alpha_{4}=O(\sqrt{\varepsilon})$ и, поскольку $\gamma_{4}(t) \leqslant \pi-\gamma_{1}(t)$, то

$$
\gamma_{4}(t)-\alpha_{4}=O(\sqrt{\varepsilon}) \quad \text { для малых } \varepsilon \quad \text { независимо от } t .
$$


По предположению $\operatorname{cur}_{3}(\alpha, \Delta(t))=\operatorname{cur}_{3}^{-}(\alpha, \Delta(t))$ :

$$
\begin{gathered}
\frac{1}{2 h_{3}(t)}\left(\operatorname{ctg} \alpha_{3}+\operatorname{ctg}\left(\gamma_{4}(t)-\alpha_{4}\right)\right)^{2} \sin ^{3} \alpha_{3}=\frac{1}{2}\left(\operatorname{ctg} \alpha_{2}+\operatorname{ctg}\left(\gamma_{3}(t)-\alpha_{3}\right)\right)^{2} \\
\times \sin ^{3}\left(\gamma_{3}(t)-\alpha_{3}\right) \\
\operatorname{ctg} \alpha_{2}+\operatorname{ctg}\left(\gamma_{3}(t)-\alpha_{3}\right)=\frac{1}{\sqrt{h_{4}(t)}}\left(\operatorname{ctg} \alpha_{3}+\operatorname{ctg}\left(\gamma_{4}(t)-\alpha_{4}\right)\right) \\
\times\left(\frac{\sin \alpha_{3}}{\sin \left(\gamma_{3}(t)-\alpha_{3}\right)}\right)^{3 / 2}, \\
\sin \alpha_{3}=\sin \gamma_{3}(t) \cos \left(\gamma_{3}(t)-\alpha_{3}\right)-\cos \gamma_{3}(t) \sin \left(\gamma_{3}(t)-\alpha_{3}\right)>\frac{\cos \left(\gamma_{3}(t)-\alpha_{3}\right)}{\sqrt{2}} \\
\left.\operatorname{ctg} \alpha_{2}>\frac{\operatorname{ctg}\left(\gamma_{3}(t)-\alpha_{3}\right)}{\sqrt{2 h_{3}(t)}\left(\operatorname{ctg} \alpha_{3}\right.}+\operatorname{ctg}\left(\gamma_{4}(t)-\alpha_{4}\right)\right)\left(\frac{\sin \alpha_{3}}{\sin \left(\gamma_{3}(t)-\alpha_{3}\right)}\right)^{1 / 2} \\
-\operatorname{ctg}\left(\gamma_{3}(t)-\alpha_{3}\right) .
\end{gathered}
$$

Из последнего неравенства и условия (2) получаем: для малых $\varepsilon$ независимо от $t$ $\operatorname{ctg} \alpha_{2}>\operatorname{ctg}\left(\gamma_{3}(t)-\alpha_{3}\right)$, что противоречит неравенствам (1).

Таким образом, в случае б) предположение $F(\alpha, t)=0$ приводит к противоречию при малых $\varepsilon$.

в) Пусть $\alpha_{2}=\varepsilon$ для некоторого $t, t \in[0 ; 1]$.

Тогда $\operatorname{cur}_{2}(\alpha, \Delta(t)) \asymp \varepsilon$ для малых $\varepsilon$ независимо от $t$. Предположим, что $\operatorname{cur}_{2}(\alpha, \Delta(t))=\operatorname{cur}_{2}^{-}(\alpha, \Delta(t))$, получим

$$
\begin{aligned}
\operatorname{cur}_{2}^{-}(\alpha, \Delta(t)) & \asymp \varepsilon, \quad \operatorname{cur}_{2}^{-}(\alpha, \Delta(t))=\frac{1}{2}\left(\operatorname{ctg} \alpha_{1}+\operatorname{tg} \varepsilon\right)^{2} \cos \varepsilon, \\
\operatorname{ctg} \alpha_{1} & \asymp \sqrt{\varepsilon}, \quad \frac{\pi}{2}-\alpha_{1} \asymp \sqrt{\varepsilon} \quad \text { для малых } \varepsilon .
\end{aligned}
$$

Из (3), в частности, следует, что при малых $\varepsilon \alpha_{1}<\pi / 2-\varepsilon$, а так как

$$
\frac{\operatorname{cur}_{1}(\alpha, \Delta(t))}{\operatorname{cur}_{2}^{-}(\alpha, \Delta(t))}=\frac{\sin ^{3} \alpha_{1}}{\sin ^{3}(\pi / 2-\varepsilon)}
$$

то

$$
\operatorname{cur}_{1}(\alpha, \Delta(t))<\operatorname{cur}_{2}^{-}(\alpha, \Delta(t)) .
$$

С другой стороны, учитьвая, что $\gamma_{1}(t)-\alpha_{1}<\pi / 2, \alpha_{4}<\pi / 2$, получаем

$$
\begin{aligned}
\operatorname{cur}_{1}^{-}(\alpha, \Delta(t)) & =\frac{1}{2 h_{4}(t)}\left(\operatorname{ctg} \alpha_{4}+\operatorname{ctg}\left(\gamma_{1}(t)-\alpha_{1}\right)\right)^{2} \sin ^{3}\left(\gamma_{1}(t)-\alpha_{1}\right) \\
& >\frac{1}{2 \sqrt{2}} \cos ^{2}\left(\gamma_{1}(t)-\alpha_{1}\right) \sin \left(\gamma_{1}(t)-\alpha_{1}\right)
\end{aligned}
$$

При малых $\varepsilon$ независимо от $t$, учитывая (3), имеем

$$
\begin{gathered}
\cos \left(\gamma_{1}(t)-\alpha_{1}\right)>\frac{1}{\sqrt{2}}, \quad \sin \left(\gamma_{1}(t)-\alpha_{1}\right)>\sin \left(\frac{\pi}{2}-\alpha_{1}\right), \\
\operatorname{cur}_{1}^{-}(\alpha, \Delta(t))>\frac{\sin \left(\pi / 2-\alpha_{1}\right)}{4 \sqrt{2}}, \quad \frac{\pi}{2}-\alpha_{1} \asymp \sqrt{\varepsilon} .
\end{gathered}
$$


Таким образом, в случае в) предположение $\operatorname{cur}_{2}(\alpha, \Delta(t))=\operatorname{cur}_{2}^{-}(\alpha, \Delta(t))$ для малых $\varepsilon$ влечет, учитывая (4) и (5), $\operatorname{cur}_{1}(\alpha, \Delta(t))<\operatorname{cur}_{1}^{-}(\alpha, \Delta(t))$, следовательно, $F(\alpha, t) \neq 0$.

Случаи $\gamma_{3}(t)-\alpha_{3}=\varepsilon, \alpha_{4}=\varepsilon, \gamma_{4}(t)-\alpha_{4}=\varepsilon$ доказываются аналогично случаю б).

Случаи $\gamma_{1}(t)-\alpha_{1}=\varepsilon, \gamma_{2}(t)-\alpha_{2}=\varepsilon, \alpha_{3}=\varepsilon$ доказываются аналогично случаю в). Лемма доказана.

ТЕОРема. Для любого выпуклого четырехугольника $f_{1} f_{2} f_{3} f_{4}$ класс $Q(\Delta)$, $\Delta=\left\{f_{1}, f_{2}, f_{3}, f_{4}\right\}$, не nycm.

ДОКАЗАТЕЛЬСТВо. Согласно принятьм обозначениям, достаточно доказать, что существует набор $\alpha^{0}$ такой, что $F\left(\alpha^{0}, 0\right)=0$. В этом случае $\alpha^{0}$ определяет кривую из класса $Q\left(\Delta^{0}\right)$, а, значит, класс $Q(\Delta)$ тоже не пуст.

Существование решения уравнения $F(\alpha, 0)=0$ доказывается посредством теоремы Лере-Шаудера [2].

Действительно, проверим выполнение условий теоремы: $F: \mathbb{R}^{5} \rightarrow \mathbb{R}^{4}, F$ определено на $\bar{\Omega}_{\varepsilon}$ - замькании области $\Omega_{\varepsilon}$, где $\Omega_{\varepsilon}=\left\{(\alpha, t) \mid \alpha \in \omega_{\varepsilon}(t), t \in[0 ; 1]\right\}$, где $\varepsilon$ удовлетворяет утверждению леммы; $F$ - непрерьвное отображение на $\bar{\Omega}_{\varepsilon} ;$ на границе области $\Omega_{\varepsilon}$, согласно лемме, уравнение $F(\alpha, t)=0$ не имеет решений; при $t=1$ уравнение $F(\alpha, 1)=0$ имеет единственное решение и степень отображения $F$ при $t=1$ отлична от нуля:

$$
\begin{aligned}
\operatorname{deg}\left[F, \omega_{\varepsilon}(1), 0\right] & =\sum_{\alpha \in F^{-1}(0) \cap \omega_{\varepsilon}(1)} \operatorname{sgn}\left[\operatorname{det} F^{\prime}(\alpha, 1)\right] \\
& =\operatorname{sgn}\left[\operatorname{det} F^{\prime}\left(\alpha^{1}, 1\right)\right]=-1,
\end{aligned}
$$

здесь $\alpha^{1}=(\pi / 4, \pi / 4, \pi / 4, \pi / 4)$.

Таким образом, все условия теоремы Лере-Шаудера выполнены и уравнение $F(\alpha, t)=0$ имеет решения для любого $t, t \in[0 ; 1]$, в частности, для $t=0$. Теорема доказана.

Московский государственный университет

Поступило

им. М.В. Ломоносова

27.05 .96

\section{СПИСОК ЦИТИРОВАННОЙ ЛИТЕРАТУРЫ}

1. Томилин А. В. // Фундам. и прикл. матем. 1996. Т. 1. С. 251-266. 2. Лерэй Ж., Шаудер Ю. // УМН. 1946. Т. 1. № 3, 4. С. 71-96. 